\title{
Ulcerative Heliotrope Rash in Antimelanoma Differentiation- Associated Gene 5 Dermatomyositis
}

\author{
T. Tyler Daugherty, MD; Justin T. Cheeley, MD
}

\section{PRACTICE POINTS}

- Antimelanoma differentiation-associated gene 5 dermatomyositis (anti-MDA5 DM) can present with an ulcerative heliotrope rash.

- Ulceration of the heliotrope rash in anti-MDA5 DM may indicate disease progression.

- Rapidly progressive interstitial lung disease is highly associated with anti-MDA5 DM.

Antimelanoma differentiation-associated gene 5 dermatomyositis (anti-MDA5 DM) is an amyopathic subtype of DM that presents with the classic cutaneous findings of DM, such as a heliotrope rash, Gottron papules, and the shawl sign, combined with mucocutaneous ulcerations. This subtype of DM also is highly associated with rapidly progressive interstitial lung disease (ILD). We report 2 cases of a heretofore novel presentation of the overlap of these cutaneous features in the form of an ulcerative heliotrope rash; furthermore, the rash was associated with progression of ILD in both cases. Finally, we review the current state of clinical care and research related to anti-MDA5 DM in terms of clinical presentations, diagnostic methods, and treatment options.

Cutis. 2021;107:E5-E8.

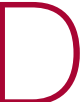
ermatomyositis (DM) is an autoimmune condition characterized by skin and muscle inflammation with an estimated incidence of 9 cases per 1 million people. The incidence of amyopathic DM, which

includes antimelanoma differentiation-associated gene 5 (anti-MDA5) DM, is approximately 2 cases per 1 million people. ${ }^{1}$ Classic cutaneous manifestations of DM include a heliotrope rash, Gottron papules, and the shawl sign. Features of anti-MDA5 DM include cutaneous ulcerations, most commonly overlying Gottron papules on the elbows and digits, as well as painful palmar macules and papules. We describe 2 patients with anti-MDA5 DM who presented with an ulcerative heliotrope rash. Although heliotrope rash is classic for DM and cutaneous ulcerations are a hallmark of the anti-MDA5 subtype of DM, overlap of these cutaneous manifestations is not commonly reported. Furthermore, ulcerations of the lateral canthi were associated with rapidly progressive interstitial lung disease (ILD).

\section{Case Reports}

Patient 1-A woman in her 30s presented with diffuse arthralgias, bilateral eyelid edema, fatigue, and a progressive diffuse exanthem of 3 months' duration. A review of systems was notable for the absence of myalgias. Physical examination revealed periorbital poikilodermatous patches with erythematous-to-violaceous plaques along the eyelid margins, violaceous papules on the dorsal knuckles, and edematous eroded plaques on the palmar fingertips. The patient was found to have a positive antinuclear antibody titer of 1:320 (reference range, $<1: 80$ ) with a speckled pattern. A computed tomography (CT) scan of the chest showed patchy bilateral ground-glass opacities that were concerning for ILD. The

From the Department of Dermatology, Emory University School of Medicine, Atlanta, Georgia. Dr. Cheeley also is from the Division of Internal Medicine and Geriatrics.

The authors report no conflict of interest

Correspondence: Justin T. Cheeley, MD, 1525 Clifton Rd NE, Dermatology Office Ste 100, Office 122, Atlanta, GA 30322 (jcheele@emory.edu). doi:10.12788/cutis.0260 
cutaneous erosions, absence of myalgias, considerable proximal weakness, radiographic evidence of ILD, and positive antinuclear antibody test were clinically suggestive of anti-MDA5 DM. Further workup confirmed this diagnosis with positive reactivity to MDA5 by line immunoassay. The patient was treated with intravenous corticosteroids and was discharged after a 17-day hospitalization; however, she presented 2 months later to outpatient dermatology for progression of the cutaneous ulcerations, at which time an ulcerative heliotrope rash (Figure 1) was identified. Despite compliance with oral corticosteroids $(1 \mathrm{mg} / \mathrm{kg} / \mathrm{d})$, she was hospitalized 1 month later for progressive respiratory insufficiency. A chest CT showed ground-glass linear opacities centrally located in all lobes of both lungs, consistent with rapidly progressive ILD. Over the course of her 5-day hospitalization, she was treated with corticosteroids, intravenous immunoglobulin (IVIG), and mycophenolate mofetil. The patient responded well to these therapies, leading to resolution of the respiratory symptoms, and she was discharged with plans to continue this regimen as an outpatient.

Patient 2-A woman in her late 30s with a history of known anti-MDA5 DM confirmed by line immunoassay 1 year prior presented to the emergency department with shortness of breath due to progressive ILD and a worsening exanthem. Dermatology was consulted to provide treatment recommendations. The treatment team was concerned for infection or anti-MDA5 DM disease progression. Physical examination revealed an ulcerative heliotrope rash (Figure 2) in addition to cutaneous findings classic for anti-MDA5 DM. Despite interventions,

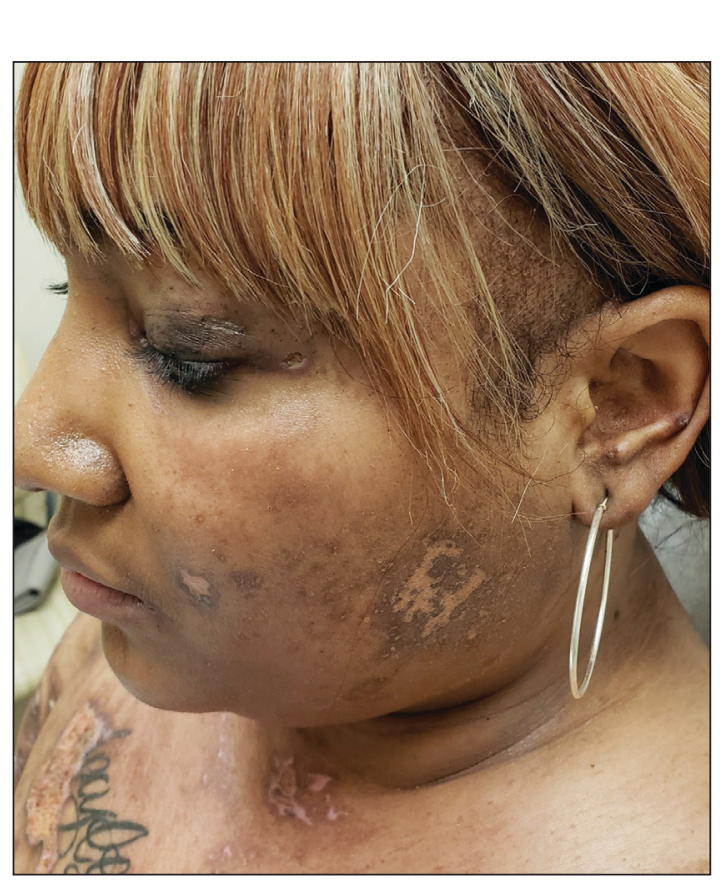

FIGURE 1. Ulcerative heliotrope rash in antimelanoma differentiationassociated gene 5 dermatomyositis. Punched-out-appearing ulcer of the left lateral canthus. including high-dose corticosteroids, rituximab, IVIG, and plasma exchange, the ILD continued to progress, and the patient and her family elected to de-escalate aggressive medical care and pursue comfort care. The patient later died in in patient hospice.

\section{Comment}

Clinical Presentation of Anti-MDA5 DM-Dermatomyositis classically presents with cutaneous manifestations including a heliotropic erythematous rash and Gottron papules as well as accompanying muscle weakness. ${ }^{2}$ However, a subtype known as amyopathic DM, which includes antiMDA5 DM, usually presents without muscle involvement. ${ }^{3}$ Clinical muscle weakness has been reported in cases of anti-MDA5 DM, though it is less likely in these patients. ${ }^{4}$ The characteristic cutaneous phenotype of anti-MDA5 DM was described by Fiorentino et $\mathrm{al}^{5}$ in 2011 through a seminal retrospective study. Kurtzman and Vleugels ${ }^{6}$ provided validation of the clinical features of anti-MDA5 DM in their 2018 review. The classic cutaneous phenotype of anti-MDA5 DM consists of tender palmar papules and/or skin ulcerations that commonly develop over Gottron papules on the knuckles and digits, lateral nail folds, and elbows. ${ }^{7-10}$ A meta-analysis of 1500 patients with anti-MDA5 DM found a statistically significant association with alopecia, Gottron sign or papules, mechanic's hands, and $\mathrm{V}$ rash $(P<.05)$, as well as skin ulcers, panniculitis, arthritis/arthralgia, pneumomediastinum, and rapidly progressive ILD (RP-ILD) $(P \leq .01){ }^{4}$ Rapidly progressive ILD is highly associated with antiMDA5 DM. ${ }^{6,11}$

While a heliotrope rash is classic for DM, and ulcerations are a hallmark of the anti-MDA5 DM subtype, overlap of these cutaneous manifestations is not commonly reported. In both cases presented here, ulcerations of the lateral canthi were associated with progression of ILD.

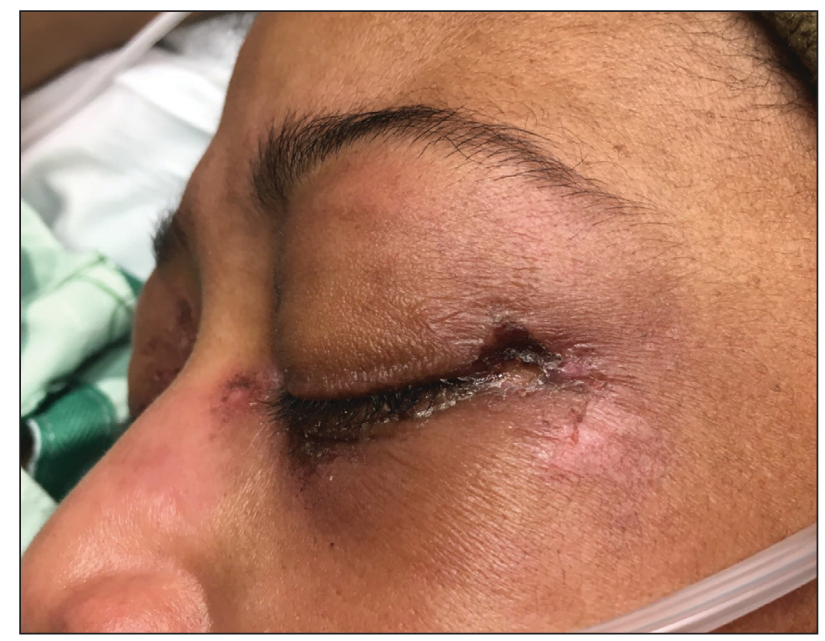

FIGURE 2. Ulcerative heliotrope rash in antimelanoma differentiationassociated gene 5 dermatomyositis. Heme-crusted ulcer of the left lateral canthus. 
Diagnosis of Anti-MDA5 DM-Anti-MDA5 DM is defined by the presence of the anti-MDA5 antibody in the serum, named for its reactivity against the RNA helicase encoded by MDA5, within the clinical context of cutaneous signs of DM as described above. ${ }^{12}$

As described by Rider et $\mathrm{al}^{13}$ a thorough laboratory analysis, including complete blood cell count, serum electrolytes, calcium, magnesium, phosphorus, and thyroidstimulating hormone, is necessary to rule out conditions with similar presentations. Additionally, serum analysis for elevated muscle enzymes (creatinine phosphokinase, aldolase, lactate dehydrogenase, alanine aminotransferase, and aspartate aminotransferase) is necessary to assess for subclinical muscle involvement. Serologic evidence of myositis usually denotes an alternative diagnosis. ${ }^{13}$ Antinuclear antibodies and myositis-specific antibody positivity are much less frequent in the antiMDA5 DM subtype than in other forms of DM. ${ }^{6}$

Anti-MDA5 antibody titer, ferritin, and IL-18 can be trended and may be useful in the evaluation of the response to treatment and ILD status in patients with anti-MDA5 DM. ${ }^{14,15}$ Elevated alveolar-arterial gradient, serum ferritin, serum chitotriosidase, and serum chitinase-3-like protein 1 (YKL-40) have each been associated with poorer prognosis of anti-MDA5 DM. The aforementioned serologies therefore may be helpful in determination of risk stratification and treatment aggressiveness. ${ }^{16-19}$

Because of its strong association with RP-ILD, screening for pulmonary disease is necessary in all patients with confirmed or strongly suspected anti-MDA5 DM. Screening can be performed with pulmonary function testing; however, high-resolution chest CT is the gold standard for diagnosis of ILD. ${ }^{20}$

Finally, all patients with a new diagnosis of DM should be evaluated for underlying malignancy through cancer screenings, given the propensity for DM to present as a paraneoplastic process. ${ }^{21}$ However, reports have indicated that the anti-MDA5 DM subtype may have a reduced risk for or an inverse relationship with underlying malignancy. ${ }^{5}$

Treatment Options for Anti-MDA5 DM-Early and aggressive therapy should be considered in the treatment of anti-MDA5 DM because of its association with RP-ILD. No treatment protocol is well established; thus, an individualized therapeutic approach may be guided by symptom severity and the clinical, radiographic, or functional evidence of ILD. ${ }^{6}$ High-dose systemic corticosteroids are first line, either in combination with or as a bridge to corticosteroid-sparing agents for immunosuppression. Many steroid-sparing medications have been employed with varying success. Mycophenolate mofetil is a reasonable first-line corticosteroid-sparing immunosuppressant agent, given its added benefit of attenuating ILD progression. ${ }^{6}$ A combination of high-dose corticosteroids, cyclosporine, and cyclophosphamide is utilized by some initially in the treatment of anti-MDA5 with
ILD. ${ }^{22,23}$ While others have used combinations of these immunomodulatory agents with mycophenolate mofetil, IVIG, rituximab, azathioprine, tofacitinib, and polymyxin $\mathrm{B}$, direct hemoperfusion has been added, leading to successful remission..$^{23-28}$

\section{Conclusion}

We present 2 patients with anti-MDA5 DM who demonstrated a rare cutaneous manifestation of an ulcerative heliotrope rash. In both cases, this cutaneous finding was associated with the development of RP-ILD. Because of the strong association with and rapid progression of ILD seen in anti-MDA5 DM, early identification and aggressive treatment of this subtype are imperative. The clinician should recognize nonacral locations of cutaneous ulcerations, including an ulcerated heliotrope rash, to optimize diagnosis and management.

\section{REFERENCES}

1. Bendewald MJ, Wetter DA, Li X, et al. Incidence of dermatomyositis and clinically amyopathic dermatomyositis: a population-based study in Olmsted County, Minnesota. Arch Dermatol. 2010;146:26-30. doi:10.1001/archdermatol.2009.328

2. Bogdanov I, Kazandjieva J, Darlenski R, et al. Dermatomyositis: current concepts. Clin Dermatol. 2018;36:450-458. doi:10.1016/j. clindermatol.2018.04.003

- Caproni M, Cardinali C, Parodi A, et al. Amyopathic dermatomyositis: a review by the Italian Group of Immunodermatology. Arch Dermatol. 2002;138:23-27. doi:10.1001/archderm.138.1.23

4. Li J, LiuY, LiY, et al. Associations between anti-melanoma differentiationassociated gene 5 antibody and demographics, clinical characteristics and laboratory results of patients with dermatomyositis: a systematic metaanalysis. J Dermatol. 2018;45:46-52. doi:10.1111/1346-8138.14092

5. Fiorentino D, Chung L, Zwerner J, et al. The mucocutaneous and systemic phenotype of dermatomyositis patients with antibodies to MDA5 (CADM-140): a retrospective study. J Am Acad Dermatol. 2011;65:25-34. doi:10.1016/j.jaad.2010.09.016

6. Kurtzman DJB, Vleugels RA. Anti-melanoma differentiation-associated gene 5 (MDA5) dermatomyositis: a concise review with an emphasis on distinctive clinical features. J Am Acad Dermatol. 2018;78:776-785. doi:10.1016/j.jaad.2017.12.010

7. Narang NS, Casciola-Rosen L, Li S, et al. Cutaneous ulceration in dermatomyositis: association with anti-melanoma differentiationassociated gene 5 antibodies and interstitial lung disease: analysis of skin ulcers in dermatomyositis. Arthritis Care Res. 2015;67:667-672. doi:10.1002/acr.22498

8. Charrow A, Vleugels RA. Cutaneous ulcerations in anti-MDA5 dermatomyositis. N Engl J Med. 2019;381:465. doi:10.1056/NEJMicm1816147

9. Cao H, Xia Q, Pan M, et al. Gottron papules and Gottron sign with ulceration: a distinctive cutaneous feature in a subset of patients with classic dermatomyositis and clinically amyopathic dermatomyositis. J Rheumatol. 2016;43:1735-1742. doi:10.3899/jrheum.160024

10. Moghadam-Kia S, Oddis CV, Sato S, et al. Antimelanoma differentiation-associated gene 5 antibody: expanding the clinical spectrum in North American patients with dermatomyositis. J Rheumatol. 2017;44:319-325. doi:10.3899/jrheum.160682

11. Li L, Wang Q, Wen $\mathrm{X}$, et al. Assessment of anti-MDA5 antibody as a diagnostic biomarker in patients with dermatomyositis-associated interstitial lung disease or rapidly progressive interstitial lung disease. Oncotarget. 2017;876129-76140. doi:10.18632/oncotarget.19050

12. Sato S, Hoshino K, Satoh T, et al. RNA helicase encoded by melanoma differentiation-associated gene 5 is a major autoantigen in patients with clinically amyopathic dermatomyositis: association with rapidly progressive interstitial lung disease. Arthritis Rheum. 2009; 60:2193-2200. doi:10.1002/art.24621 
13. Rider LG, Miller FW. Deciphering the clinical presentations, pathogenesis, and treatment of the idiopathic inflammatory myopathies. JAMA. 2011;305:183-190. doi:10.1001/jama.2010.1977

14. Nishioka A, Tsunoda S, Abe T, et al. Serum neopterin as well as ferritin, soluble interleukin-2 receptor, KL-6 and anti-MDA5 antibody titer provide markers of the response to therapy in patients with interstitial lung disease complicating anti-MDA5 antibody-positive dermatomyositis. Mod Rheumatol. 2019;29:814-820. doi:10.1080/14397595.2018.1548918

15. Gono T, Sato S, Kawaguchi Y, et al. Anti-MDA5 antibody, ferritin and IL-18 are useful for the evaluation of response to treatment in interstitial lung disease with anti-MDA5 antibody-positive dermatomyositis. Rheumatology. 2012;51:1563-1570. doi:10.1093/rheumatology/kes102

16. Jiang L, Wang Y, Peng Q, et al. Serum YKL-40 level is associated with severity of interstitial lung disease and poor prognosis in dermatomyositis with anti-MDA5 antibody. Clin Rheumatol. 2019;38:1655-1663. doi:10.1007/s10067-019-04457-w

17. Fujisawa T, Hozumi H, Yasui $H$, et al. Clinical significance of serum chitotriosidase level in anti-MDA5 antibody-positive dermatomyositisassociated interstitial lung disease. J Rheumatol. 2019;46:935-942. doi:10.3899/jrheum.180825

18. Enomoto N, Oyama Y, Enomoto Y, et al. Prognostic evaluation of serum ferritin in acute exacerbation of idiopathic pulmonary fibrosis. Clin Resp J. 2018;12:2378-2389. doi:10.1111/crj.12918

19. Fujiki Y, Kotani T, Isoda K, et al. Evaluation of clinical prognostic factors for interstitial pneumonia in anti-MDA5 antibody-positive dermatomyositis patients. Mod Rheumatol. 2018;28:133-140. doi:10.1080/14397 595.2017.1318468

20. Raghu G, Remy-Jardin M, Myers JL, et al; American Thoracic Society, European Respiratory Society, Japanese Respiratory Society, and Latin American Thoracic Society. Diagnosis of idiopathic pulmonary fibrosis. an official ATS/ERS/JRS/ALAT clinical practice guideline. Am J Respir Crit Care Med. 2018;198:E44-E68. doi:10.1164/rccm.201807-1255ST
21. Yang Z, Lin F, Qin B, et al. Polymyositis/dermatomyositis and malignancy risk: a metaanalysis study. J Rheumatol. 2015;42:282-291. doi:10.3899/jrheum.140566

22. Hisanaga J, Kotani T, Fujiki Y, et al. Successful multi-target therapy including rituximab and mycophenolate mofetil in anti-melanoma differentiation-associated gene 5 antibody-positive rapidly progressive interstitial lung disease with clinically amyopathic dermatomyositis. Int J Rheumatic Dis. 2017;20:2182-2185. doi:10.1111/1756-185X.13136

23. Kameda H, Nagasawa H, Ogawa H, et al. Combination therapy with corticosteroids, cyclosporin A, and intravenous pulse cyclophosphamide for acute/subacute interstitial pneumonia in patients with dermatomyositis. J Rheumatol. 2005;32:1719-1726.

24. EndoY, Koga T, Suzuki T, et al. Successful treatment of plasma exchange for rapidly progressive interstitial lung disease with anti-MDA5 antibody-positive dermatomyositis: a case report. Medicine. 2018;97:e0436. doi:10.1097/MD.0000000000010436

25. So H, Wong VTL, Lao VWN, et al. Rituximab for refractory rapidly progressive interstitial lung disease related to anti-MDA5 antibodypositive amyopathic dermatomyositis. Clin Rheumatol. 2018;37:19831989. doi:10.1007/s10067-018-4122-2

26. Kurasawa K, Arai S, NamikiY, et al. Tofacitinib for refractory interstitial lung diseases in anti-melanoma differentiation-associated 5 gene antibody-positive dermatomyositis. Rheumatology. 2018;57:2114-2119. doi:10.1093/rheumatology/key188

27. Nawata T, Kubo M, Okuda S, et al. Successful treatment with intravenous cyclophosphamide for anti-melanoma differentiation-associated gene 5 antibody-positive dermatomyositis associated with myelodysplastic syndrome. Scand J Rheumatol. 2017;46:496-498. doi:10.1080/0300 9742.2016.1253770

28. Griger Z, Nagy-Vincze M, Dankó K. Pharmacological management of dermatomyositis. Exp Rev Clin Pharmacol. 2017;10:1109-1118. doi:10.10 80/17512433.2017.1353910 